\title{
Sensor Network and GeoSimulation: Keystones for Spatial Decision Support Systems
}

\author{
Nafaâ Jabeur ${ }^{1}$, Nabil Sahli ${ }^{1}$ and Hedi Haddad ${ }^{2}$ \\ ${ }^{1}$ Dhofar University \\ ${ }^{2}$ Laval University \\ ${ }^{1}$ Oman \\ ${ }^{2}$ Canada
}

\section{Introduction}

Natural hazards and man-made disasters are victimizing large numbers of people and causing significant social and economical losses. By developing efficient Spatial Decision Support Systems (SDSS), managers will be efficiently assisted in identifying and managing impending hazards. This goal could not be reached without addressing significant challenges, including data collection, management, discovery, translation, integration, visualization, and communication. As an emergent technology, sensor networks have proven efficiency in providing geoinformation for any decision support system particularly those aiming to manage hazardous events. Thanks to their spatially distributed nature, these networks could be largely deployed to collect, analyze, and communicate valuable in-situ spatial information in a timely fashion. Since some decisions are expected to be taken onthe-fly, the right data must be collected by the right set of sensors at the right time. In addition to saving the limited resources of sensor networks, this will speed up the usability of data especially if this data is provided in the right format. In order to boost the decision support process, a thorough understanding and use of the semantics of available and collected heterogeneous data will obviously help to determine what data to use and how confident one can be in the results ultimately. An appropriate representation of the geoinformation should enhance this process.

Data collected by sensors is often associated with spatial information, which makes it voluminous and difficult to assimilate by human being. In critical situations, the hazard manager has to work under pressure. Coping with such collected data is a demanding task and may increase the risk of human error. In this context, Geosimulation emerges as an efficient tool. Indeed, mapping the collected data into a simulated environment which takes into account the spatial dimension may dramatically help the hazard manager to easily visualize the correlation between data collected by sensors and the geospatial constraints.

In this chapter, we first present fundamental concepts of SDSS and the most important challenges related to their development. Second, we outline the sensor network technology as an emergent tool for leveraging SDSS. Third, we present the Geosimulation approach as another keystone to enhance SDSS. In this part, we summarize the current opportunities, research challenges, and potential benefits of this technique in SDSS. Finally, for better efficiency, we propose an encoding that emphasizes the semantics of available data and 
tracks events/effects propagation. Based upon conceptual graphs, this encoding will be used to increase the benefits from sensor networks and geosimulation in SDSS.

\section{Spatial decision support systems}

Literature about spatial decision support systems (SDSS) is abundant and covering all their facets is beyond the scope of this chapter. In this section we only introduce the main characteristics of these systems and some important challenges related to their development.

\subsection{Definition and importance of SDSS}

The concept of spatial decision support systems has emerged by the end of 1980's from the integration of two technologies: decision support systems (DSS) and geographic information systems (GIS) (Sugumaran and Sugumaran, 2005). Beyond the broad definition that SDSS are DSS designed to support decision-makers solving complex spatial problems, in the literature there is no consensus about what SDSS exactly are. Keenan (Keenan, 2003) attributed the disagreement on the definition of SDSS to the fact that DSS themselves are not clearly defined. Consequently, in the literature SDSS are often defined by their common characteristics rather than by what they exactly are. For example, Goel (Goel, 1999) listed the following common traits characterizing SDSS: they are designed to solve ill-structured problems, they have user interfaces, they combine models and data and they contain tools allowing users to explore and evaluate candidate solutions. They are supposed to provide an iterative problem-solving environment. However, we only develop the following main characteristics:

\section{Class of problems}

Malczewski (Malczewski, 1999) defined a SDSS as "an interactive computer based system designed to support a user or group of users in achieving a higher effectiveness of decisionmaking while solving a semi-structured spatial decision problem". Spatial decision problems are often ill- or semi-structured. They are multidimensional, characterised by uncertainty (which is inherent to spatial data), have decision objectives and factors that can not be fully and formally specified and do not have a unique evident solution (Densham, 1991; Gao et al., 2004; Ademiluyi and Otun, 2009). Consequently, spatial decision-making processes are usually based on the "what-if" analysis: the decision-maker defines the problem, collects and analyses data and evaluates the consequences of several alternatives (scenarios) in order to select the best one (Ademiluyi and Otun, 2009). Moreover, spatial decisions require the implication of several stakeholders; they are iterative, interactive and participative processes (Densham and Goodchild, 1989; Goel, 1999). In addition, several studies have demonstrated that human beings have several cognitive limits when reasoning about spatial data (Kahneman and Tversky, 1982). For all of these reasons, spatial decisions are inherently complex and it is very helpful to have SDSS that support them.

\section{Main components}

Decision support systems are often developed based on the "DDM paradigm", i.e., a DSS should provide capabilities related to dialog, data and modeling (Sprague and Watson, 1996). SDSS are complex systems that integrate multidisciplinary technologies, but as an extension of DSS, they should have architectural components that balance among those three capabilities (Ademiluyi and Otun, 2009). Although the number and exact description of 
components mentioned in the SDSS literature differs (see (Sugumaran and Degroote, 2010) for more details), a SDSS should at least integrate the following capabilities (Densham, 1991; Densham and Goodchild, 1989; Keenan, 2003):

1. Spatial and non spatial data management capabilities (SDBMS component): include tools supporting the collection and storage of data, the translation between different data models and data structures, and retrieving data from storage.

2. Analytical and spatial modeling capabilities (Modeling and analysis component): provide decision-makers with access to a variety of spatial models in order to help them in the decision-making process. Examples of models include statistical, mathematical, multi-criteria evaluation, cellular automata and agent-based models (Sugumaran and Degroote, 2010).

3. Spatial display and report generation capabilities (User interface component): support the visualization of the data sets and the output of models using several formats such as maps, graphics and tables.

\section{Domain specific}

Like DSS, SDSS are often domain- and problem specific, although there are some SDSS that are designed to be generic (Sugumaran and Degroote, 2010). SDSS have been applied to several domains, such as urban planning, environment and natural resource management, transportation and business, which led to the use of several spatial modeling techniques and technologies. Reusing modeling techniques is not always evident, and the development of SDSS is often complex, expensive and requires the acquisition of specific domain or problem knowledge.

\subsection{SDSS vs. GIS}

Although GIS offer spatial data management and analysis capabilities that are attributes of SDSS, they are usually not considered as SDSS (Sugumaran and Degroote, 2010) The major reasons are that GIS lack of analytical modeling capabilities and techniques for effective scenarios evaluation (Segrera et al., 2003). In addition, Malczewski (Malczewski, 1999) pointed out that GIS do not usually have tools for presenting and evaluating choices with conflicting multi-criteria and goals. These deficiencies limit the effectiveness of GIS in supporting complex spatial decision problems (Densham, 1991). SDSS are thus usually developed based on GIS software coupled with additional modeling and analysis techniques such as multi-criteria decision analysis (MCDA), artificial intelligence, agentbased modeling and simulation techniques.

\subsection{Challenges for SDSS}

Although SDSS have reached the maturity stage and have been successfully implemented in several domains, there are still several challenges to be addressed. Sugumaran and Degroote (Sugumaran and Degroote, 2010) identified technical, technological, social and educational challenges, but in the following we only focus on some important challenges:

1. As we previously mentioned, an important capability of any SDSS is the presentation of output and scenario alternatives to users. Evers (Evers, 2007) listed the lack of capabilities for evaluating alternatives as a major reason for the inefficacity of SDSS. Uran and Janssen (Uran and Janssen, 2003) indicated that an SDSS must allow the user creating and testing alternatives using intuitive and simple techniques for end users. In addition, it is very important for the decision-maker to be provided with the relevant 
information, and any lack or over-load could potentially limit her ability to make good informed decisions. Providing the relevant and meaningful information is an important challenge. Most used techniques are visual (maps, 3D visualisations, etc.) or quantitative (tables, graphs, etc.). There is currently a lack of qualitative techniques that allow the decision-maker exploring the consequences of her alternative solutions with a simple and meaningful language.

2. Developing SDSS for environmental monitoring and crisis situations requires not only the capability of modeling dynamic spatial phenomena, but also the ability to update those models with real-time data. Moreover, the system must provide an output that is relevant and easy to understand to the non-technical decision-maker, because any misleading or ambiguity can have to catastrophic consequences. However, GIS traditionally do not handle dynamic spatial models, and in order to remedy this limit, they have been coupled with modeling techniques from several research fields, especially cellular automata and agent-based simulations. Although this has led to the emergence of Dynamic GIS (Albrecht, 2007), dynamic spatial knowledge representation and modeling is an active research field (Haddad and Moulin, 2010a), and the lack of standards and efficient representation formalisms makes challenging the development of SDSS for real-time or near-real time spatial problems.

3. Decision-makers often need to examine various situations simultaneously at different levels of detail (macro-, meso- and micro- scales of representations). This is an important issue since the modeled phenomena and observed patterns may be different from one level of detail to another, and since interferences may arise between phenomena developing in different levels of detail. The level of detail is very important and complex problem, because it does not only require modeling the problem's dimensions at different spatial and temporal levels of details but also linking these different levels of detail.

4. Most SDSS were developed independently of one another, and with the development of internet and web-based SDSS (Sugumaran and Sugumaran, 2005), there is more and more a need for sharing and accessing spatial data from several distributed and heterogeneous sources. Interoperability is then an important challenge to be addressed. The Open Geospatial Consortium (OGC) has been developing different interoperability standards for web applications, including Web Map Service (WMS) and Web Feature Service (WFS), and the issue of developing SDSS using these standards has been recently raised (Zhang, 2010). In addition, semantic interoperability is an active research field in geographic anthologies and semantic web (Wang et al., 2007), and the lack of standards makes the development and deployment of ontology-driven SDSS difficult.

In the remaining of this chapter we focus on SDSS for dynamic environmental monitoring and crisis response and we present an approach based on sensor networks and geosimulation techniques to address their related challenges.

\section{Sensor network-based SDSS}

\subsection{Survey on sensor network-based SDSS}

A SDSS embodies geomatic principles for situating decision-making in space. It often uses a GIS component to provide spatial analysis functionality (McCarthy et al., 2008). In a variety of applications, updated spatio-temporal data on current conditions in the environment of interest is decisive in making appropriate decisions. This data could be collected from the field using existing techniques, such as sensors, satellites, sonar, and radars. 
Sensors are small devices, deployed in an environment for the purpose of collecting data for specific needs, such as monitoring temperature, pressure, moisture, motion, vibration, or gas or tracking objects. Since the energy and processing constrained sensors are frequently prone to failure, robbery, and destruction, they are unable to achieve their tasks individually. For this reason, they are deployed within an extended infrastructure called a sensor network. In this infrastructure, the spatially distributed sensors could implement complex behaviors and reach their common goals through a collaborative effort. Thanks to this collaboration, they are able to collect important spatio-temporal data for a variety of applications. This quality motivates the link between SDSS and sensor networks, especially when data is needed to be collected remotely and in real-time with tools which can operate in harsh environments.

In the literature, several works have proposed sensor network-based SDSS. In the oil and gas industry, Supervisory Control \& Data Acquisition Systems (SCADA) are using sensors in the monitoring of the processes within a total operating environment. The sensors are connected throughout an oil refinery or pipeline network, each providing a continuous flow of information and data about the operating processes. Chien and his colleagues (Chien et al., 2010) have deployed an integrated space in-situ sensor network for monitoring volcanic activity. In addition to ground sensors deployed to the Mount Saint Helens volcano, this network includes a spacecraft for earth observation. The information collected with sensors is integrated with data collected with an intelligent network of "spider" instrument deployed to the surface of the Mount Saint Helens volcano. The resulting data are used by hybrid manual reporting systems such as the Volcanic Ash Advisory Centers (VAAC) and Air Force Weather Advisory System (AFWA). Within the same context, Song and his colleagues (Song et al., 2008) have proposed OASIS. OASIS is a prototype system that aims at providing scientists and decision-makers with a tool composed of a "smart" ground sensor network integrated with "smart" space-borne remote sensing assets to enable prompt assessments of rapidly evolving geophysical events in a volcanic environment. The system constantly acquires and analyzes both geophysical and system operational data and makes autonomous decisions and actions to optimize data collection based on scientific priorities and network capabilities. The data collected by OASIS is also made available to a team of scientists for interactive analysis in real-time.

$\mathrm{O}^{\prime}$ Brien and his colleagues (O'Brien et al., 2009) have presented a distributed software architecture enabling decision support in dynamic ad hoc sensor networks, which enables rapid and robust implementation of an intelligent jobsite. The architecture includes three layers: a layer for expressive approachable decision support application development, a layer for expressive data processing, and a layer for efficient sensor communication. According to the authors, the implemented prototype can speed up application development by reducing the need for domain developers to have detailed knowledge about device specifications. It can also increase the reusability of software and protocols developed at all levels and make the architecture applicable to other industries than construction.

Wicklet and Potter (Wicklet and Potter, 2009) have presented three steps for informationgathering, from sensor data to decision support. These steps are: data validation, data aggregation and abstraction, and information interpretation. Ling and his colleagues (Ling et al., 2007) have proposed a sparse undersea sensor network decision support system based on spatial and temporal random field. The system has been presented as suitable for multiple targets detection and tracking. In this system, an optimization based random field 
estimation method has been developed to characterize spatially distributed sensor reports without making any assumptions on their underlying statistical distributions.

In (James et al., 2008), the authors have proposed an approach that aims at performing information-centric analysis within a GIS-based decision support environment using expert knowledge. By shifting towards a more information-centric approach to collect and use sensor measurements, more advanced analysis techniques can be applied. These techniques can make use of stored data about not only sensor measurements and what they represent, but also about how they were collected and the spatial context related to them. In a related work, Rozic (Rozic, 2006) has proposed REASON, which is a Spatial Decision Support Framework that uses an ontology-based approach in order to interpret many different types of data provided by sensor measurements. In such systems, binding and transforming the sensor data into timely information which is relevant to the problem is a challenging task. In order to solve this issue, we have proposed in a related work (Jabeur and Haddad, 2009) to allow sensors to autonomously cooperate and coordinate their efforts while emphasising on data semantics. By encoding causality relationships about natural phenomena and their effects in time and space with the formalism of conceptual graphs, we have proposed an approach that implements a progressive management of hazardous events. This approach would provide the decision-makers with timely valuable information on hazardous events of interest, such as floods and volcano eruption.

In (Tamayo et al., 2010), the authors have conducted the design and implementation of a decision-support system for monitoring crops using wireless sensor networks. The prototype implemented includes tools that provide real-time information about the crop status, surrounding environment and potential risks such as pests and diseases.

In (Filippoupolitis and Gelenbe, 2009), the authors have proposed a distributed system that computes the best evacuation routes in real-time, while a hazard is spreading inside a building. The system includes a network of decision nodes and sensor nodes, positioned in specific locations inside the building. The recommendations of the decision nodes are computed in a distributed manner then communicated to evacuate or rescue people located in the vicinity.

In order to support rain-fed agriculture in India, Jacques and his colleagues (Jacques et al., 2007) have proposed COMMON-Sense Net system, which aims at providing farmers with environment data. This system can provide farmers with valuable data allowing them to improve the production of semiarid regions in a cluster of villages in Southern Karnataka.

Mastering a ship in heavy seas is always a challenge, especially in night time. The officer of the watch has to "sense" the weather and waves in order to preserve the cargo and navigate the ship without undue motions, stress or strains. SeaSense (Nielsen, 2004) is designed to support the natural human senses in this respect by providing an accurate estimation of the actual significant wave height, the wave period, and the wave direction. Furthermore, and based on the measurements and estimations of sea state, SeaSense provides actual information on the sea-keeping performance of the ship and offers decision support on how to operate the ship within acceptable limits.

\subsection{Opportunities and challenges in sensor network-based SDSS}

Any SDSS should provide decision-makers with the right data, at the right time, from the right spatial location. In addition, the SDSS must allow the decision-makers to have a global view about what is happening in the application environment. As this environment may be large-scale, harsh, and likely impossible for human to access, the requested data could only 
be collected remotely. There are several existing techniques that can help in achieving this task, such as radars, satellites, and sensors. Depending on the availability of resources and the application context, one or more technologies can be deployed.

Sensor networks afford SDSSs with opportunities along five axes (Fig. 1):

1. For which goals data are collected: The sensor network should collect the requested data depending on the goals of the application. For example, in a flood scenario, sensors should collect data on the level and movement of water, the conditions of soil slope, and water pollution. Moreover, sensors can deliver to the SDSS data in several formats including those that are compatible with widely used standards.

2. When data will be collected: sensors should be activated at the right time to collect and deliver the requested data to decision-makers through the SDSS.

3. Where data will be collected: sensors should collect data from the right location and thus decision-makers could have updated information on locations of interest.

4. Who will collect data: sensors should collect the requested data depending on their current locations. If they are not explicitly appointed by the decision-makers, they should self-organize in order to identify the subset of sensors that will be in charge of collecting the requested data.

5. How data will be collected: sensors generally follow sleep/wakeup cycles. They are programmed to collect data according to predefined schedules. Depending on the current situation, this schedule has to be adapted in order to collect data at appropriate times. Moreover, data can be collected through a collaborative effort from several sensors.

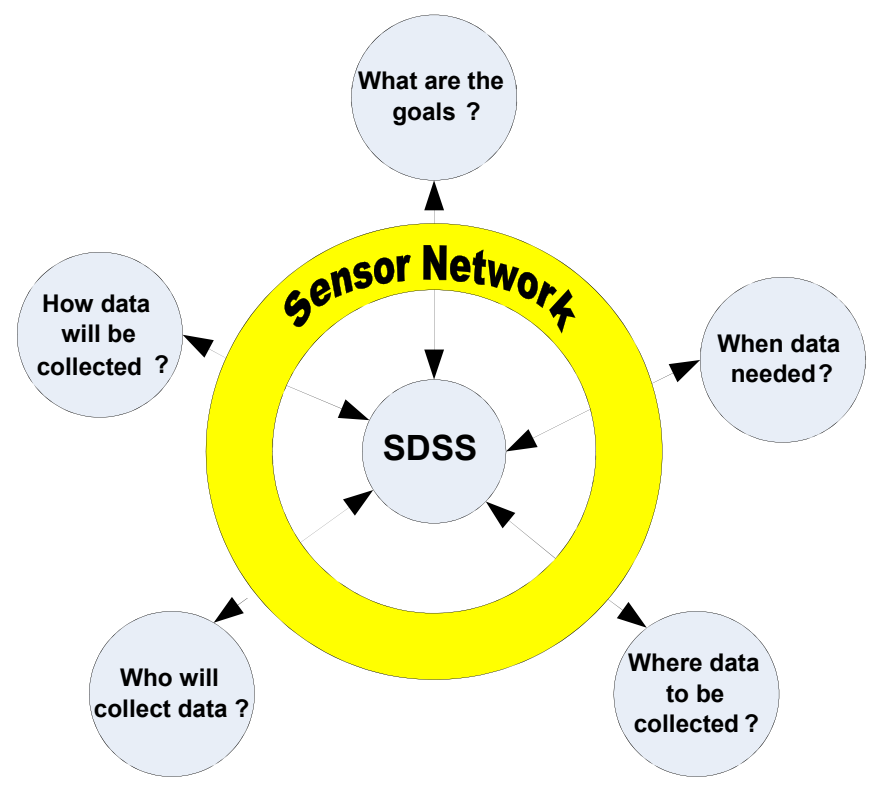

Fig. 1. Opportunities in sensor network-based SDSS

As sensors are commonly characterized with their limited processing capabilities and shortbattery lives, they are commonly prone to failure and have to save their energy by following 
sleep/wakeup cycles. In addition, due to the characteristics of operating environments, the sensors can be destructed, lost, and even stolen. Consequently, the topology of the sensor network is frequently changing and thus pulling data from the network could be affected. This affects the fluent feeding of the SDSS with the requested data. As such, this represents the main challenge of binding SDSS with sensor networks.

Binding SDSS and sensor networks concerns several issues, including:

1. Communication: as sensors are generally spatially distributed and do not maintain reliable communication pathways, the exchange of data between the SDSS and the sensor network should not be straightforward. Thus, data should not be pulled out appropriately from the network.

2. Data format: the Open Geospatial Consortium's Sensor Web Enablement (Botts et al., 2006) working group has created an entire suite of standards related to sensor networks. In this context, SensorML could be used to describe sensors and sensor platforms, O\&M (an XML schema which complements SensorML) could be used to describe measurements made by sensors, and SOS (Na and Priest, 2006) can make use of SensorML and O\&M to provide an interactive, useful view of current and historical conditions at a site. In spite of such standards, some SDSSs may use proprietary data formats that may cause some incompatibility with sensor data.

3. Synchronization: in some real-time applications, communication between the sensor network and the SDSS must be in timely fashion. This could not be guaranteed all the time, particularly due to sensor network constraints.

\section{GeoSimulation-based SDSS for crisis response}

Hardly a day passes without some form of crisis, disaster or tragedy hitting the headlines. At the same time, new technologies have made great steps, which, in theory at least, have been accomplished for the safety of human beings and the environment. Unfortunately, many technological achievements are responsible for most of the disasters occurring today. Paradoxically, we need technology to better deal with these disasters when they occur.

When problems are well-structured and relatively static (e.g., resource allocation), mathematical programming methods can be built into the SDSS. For dynamic situations, such as crisis management, simulation is a better tool (Fedra and Reitsma, 1990; Zhang and Grant, 1993). In this section we first make an overview of SDSS used for crisis response with a particular emphasis on those based on geo-simulation. We then enumerate the main challenges and opportunities of geosimulation-based SDSS for crisis response.

\subsection{Review}

SDSSs have been successful in addressing the spatial component of natural and technological hazards (Fedra, 1997). As a result, several specialized SDSS have been developed that assist decision-makers with hazards. For instance, regarding wildfires, a multidisciplinary system has been developed by Keramitsoglou and his colleagues (Keramitsoglouet al., 2009) to provide rational and quantitative information based on the site-specific circumstances and the possible consequences. The system's architecture consists of several distinct supplementary modules of near real-time satellite monitoring and fire forecast using an integrated framework of satellite Remote Sensing, GIS, and RDBMS technologies equipped with interactive communication capabilities. The system may handle multiple fire ignitions and support decisions regarding dispatching of utilities, equipment, and personnel that would appropriately attack the fire front. 
Garbolino and his colleagues (Garbolino et al., 2007) proposed Computer-Aided Management of Emergency Operations (CAMEO) which is a SDSS for assessing the impact of a gas release from a hazardous material transportation accident on the population in a dense urbanized area. The SDSS uses a database that centralizes, in real time, the data on hazardous material transportation coming from both embarked sensors monitoring the physical conditions of the material in the tank of the truck, the truck route, its speed etc. and an installed optical sensor placed in a highway station which detects orange plates of the hazardous material trucks crossing the highway station. CAMEO is associated to the GIS platform in order to integrate the databases on the stakes of the territory (population, housing, companies, etc.) with the maps of the atmospheric dispersion distances of toxics coming from the simulations computed with CAMEO. The overlapping of the maps of these gas release distances and the data on the stakes allow the user to identify the exposed (or impacted) stakes.

Many other SDSS were developed for droughts and agricultural pestilence (MacLean et al., 2000; Agatsiva and Oroda, 2002), floods, hurricanes, wildfires, and earthquakes (Fulcher et al., 1995; Radke, 1995; Sanders and Tabuchi, 2000; Jaber et al., 2001; Hodgson and Cutter, 2002; Jensen and Hodgson, 2006). Most of these SDSS were designed to help human decision-makers to take the right decisions. However, more sophisticated SDSS can also assist these decision-makers in planning interventions during crisis situations. These SDDS are called geosimulation-based SDSS as they use geosimulation to support human planners. To better understand these SDSS, we discuss the necessity of the geosimulation process in what follows.

Human planning requires the simulation of plans, which is itself based on anticipation and schematisation (Hoc, 1987). When facing a new situation, a human being is able to detect similarities with well-known situations and to anticipate events or properties which are not yet totally identified (Hoc, 1987) thanks to his sense of anticipation. According to Craik (Craik, 1946), planning is a very refined activity implying a mental formulation of future states of the world in order to simulate the real world behaviour and then to predict future events. However, human planning capabilities are limited. In (Kahneman and Tversky, 1982), the authors claim that simulation is difficult for humans, which is proven by Forbus' experiences (Borbus, 1981) which show that simulation cannot be done by humans, expect in trivial cases.

Planning becomes more complex when addressing uncertain situations such as during crisis response interventions. In such cases, accurate predictions about plan executions remain a hard task for human planners. The AI community proposed solutions such as the Simulation-Based Planning approach SBP which consists in associating planning and simulation. Each generated plan is simulated in order to be tested and evaluated. The most appropriate plan is kept. As we focus on crisis situations, the spatial component is crucial which makes the problem even more complex.

In order to plan within a large-scale space, a person relies on a sophisticated mechanism based on cognitive maps which are mental constructs that we use to understand and know environments and use to make spatial decisions (Kitchin, 1994). Despite its sophistication, it remains very limited as many errors, mainly metrical, can occur. Sources of these metrical errors include distortion, distances, and directions. Consequently, and despite his exceptional cognitive capabilities, a human being has several limitations when trying to plan in a real large-scale geographic space. An SDSS based on a geosimulation (simulation using GIS data) approach would help overcome some of these limitations. Simulation is even more 
important for emergency planning. Indeed, when based on realistic assumptions, simulation offers an efficient way of modeling crisis response tasks.

In the domain of forest firefighting for example, we developed a geosimulation-based SDSS which relies on a four-layer architecture (Sahli and Moulin, 2009; Sahli and Jabeur, 2011). An adaptation to this architecture is discussed later (see Subsection 4.3). In the air navigation domain, (Ozan and Kauffmann, 2002) developed a practical tool for tactical and strategic decision-making in aircraft-based meteorological data collection called TAMDAR (Tropospheric Airborne Meteorological Data Reporting). This onboard system gathers meteorological data (using airborne sensors) as aircraft fly in the troposphere and transmit this data to the National Weather Service. Collected data will help decision-makers to process different operational alternatives (by conducting various what-if analyses using a GIS-based user interface) and to determine the best strategy for system operation. The SDSS is composed of a customized simulation-optimization engine, a data utility estimator, a GISbased analysis layer, and a user interface. They can also conduct various what-if analyses effectively by using GIS-based user interface.

In the domain of civilian evacuation, and as evacuees move, a specialized SDSS can continuously track evacuees using their current spatial location coordinate. In this context, Nisha de Silva designed a prototype of a geosimulation-based SDSS named CEMPS (Configurable Evacuation Management and Planning Simulator) (Nisha de Silva, 2000). The aim was to produce an interactive planning tool to produce simple scenarios wherein emergency planners are able to watch a simulation proceed and provide limited interaction to obtain information on the progress of the evacuation. CEMPS integrates a dynamic and interactive evacuation simulation model with a GIS which defines the terrain, road network, and related geographical elements such as the hazard source and shelters, as well as the population to be evacuated.

\subsection{Challenges and opportunities for GeoSimulation-based SDSS}

In the following, we briefly enumerate the main challenges that geosimulation-based SDSS is facing as well as the opportunities that it represents.

1. Simulation models are difficult to integrate in the SDSS: In many crisis domains, experts have done a lot of effort on model development. The difficulty in developing the DSS is not then a lack of available simulation models but rather making these models available to decision-makers (Muller et al., 2003). These simulation models are usually used in research labs without being integrated into the decision-making process. Muller and his colleagues (Muller et al., 2003) explained this gap by the following reasons: data requirements are usually only attained in a research setting; models are complex and assumptions are not well understood by managers; and deriving model input parameters is extremely time consuming and difficult.

2. Multi-disciplinary problem: To integrate simulation models in SDSS, one needs expertise in database management system, geographic information systems, computer operating systems, remote sensing and Internet searching for data gathering, graphics, as well as specific domain knowledge. Unfortunately, only few professionals/researchers have all these skills. Cross disciplinary research groups have to be set and collaborate more in order to achieve better geosimulation-based SDSS.

3. Granularity: The accuracy of predicting entities (resources and actors within the environment) behavior depends on whether the SDSS simulator uses a micro, meso, or 
macro modeling approach. The choice among the three levels of granularity depends on the trade-offs that must be made in order to maintain realistic computing power when processing large amounts of data.

4. Validation of simulated models: A simulator is intended to mimic a real-world system using actual data associated with that system. It uses assumptions that generally simplify the complex behavior of the real-world. When the SDSS simulates a highly dynamic environment as during crisis response, it is a big challenge to validate the simulation models in use.

5. Integrating data reported by citizen: Many governments are implementing large-scale distributed SDSS to incorporate data from a variety of emergency agencies in order to produce real-time "common operational pictures" of crisis situations. This goal remains a very hard task. In this context, social networks have the potential to transform emergency response by providing SDSS with data collected by citizens. Individuals are often in the best position to produce immediate, empirical and real-time observance of events simply because they are there. Pictures or videos taken by citizens from a cell phone can provide invaluable information. Integrating these data and media generated by citizens and other non-state actors with the main SDSS can enhance governmental response to crisis. This will not only imply reviewing the command and control strategies and the communication infrastructure, but also opening new research avenues on how to collect, filter, and integrate these new data into the SDSS.

5. Including a full Emergency Management Cycle: The emergency management community has long recognized that society's response to disaster events evolve through time. The succession of emergency response stages after a disaster event, known as the Emergency Management Cycle, includes a phase of response/rescue operations, a later phase of recovery/reconstruction, and a stage focused on mitigation and monitoring for future events (Cutter, 1993). Most of existing SDSSs only focus on one stage. It would be a considerable improvement if SDSS can address all stages involved in emergency response.

6. Web-based SDSS: As with other applications deployed via the web, the Internet based SDSS provides advantages over traditional desktop applications. First, the application is centrally located, simplifying distribution and maintenance. In addition, the Internet based approach increases the user base by reducing costs of access to users. However, Internet standards have some limitations for use in spatial applications, but new software and plugins continue to be developed. Current applications offer map display, but frequently fall short of providing comprehensive GIS functionality. Future developments offer the possibility of a distributed SDSS that could connect with datasets held at distant locations on the Internet.

\subsection{Combining sensors and GeoSimulation-based SDSS}

When facing a natural disaster or a crisis situation, decision-makers need to monitor the situation and then plan interventions (evacuation, rescue, etc.) while taking into account the constraints of the real word (real large-scale, dynamic, and uncertain environment).Sensors are of course one of the possible means of monitoring the environment. However, a 'deploy and ignore' approach of sensors is not appropriate in this context. In remote areas, where human interventions cannot be provided appropriately, sensors must have reasonable intelligence and autonomy to reconnoiter their surroundings, respond to changing 
environmental conditions, cope with frequently changing communication pathways and network topology, and carry out automated diagnosis and recovery.

Static sensors are used to monitor the environment and send sensed data to the SDSS, which may use this data in different ways. For example it can map the data (usually after treatment) on the geo-referenced map (Sahli and Moulin, 2009). A human user can visualise the data before taking any decision. The sensed data can also be used to feed a simulated model (within the SDSS) of the event occurring in the dynamic environment. We have already followed this approach for the forest fire problem in (Sahli and Moulin, 2009) and the train derailment problem (caused by rock falls) in (Sahli et al., 2008).

In this section we are going to focus on another type of sensors which is very useful during crisis response but much more difficult to manage. These are mobile sensors (whether autonomously or embedded in moving vehicles). They offer more flexibilities and opportunities for data acquisition and tracking events of interest in a highly dynamic environment. However, connectivity and coverage among the network components have to be maintained. To this end, it is important to prevent mobile nodes to move freely, anywhere and anytime, creating communication holes. Holes can also result from the absence of communication connectivity (sleep, robbery, or shutdown of some sensors) as well as from heavy network activities. The goal of maintaining a WSN without communication holes cannot be always guaranteed due to simultaneous movements, sleep/shutdown of some nodes, or jamming factors.

In the particular cases of natural disaster (forest fire, oil slick, flood, etc.) or crisis situations, mobile sensors have to deal with the constraints of the real world (real large-scale environment, dynamism, and uncertainty). The "real large-scale environment" constraint implies that the geographical aspects of the terrain could affect the sensors move. For example, moving a sensor in a forest in fire is not always possible as movements depend on the vegetation and the natural obstacles in the surrounding geographic space. The "uncertainty" and "dynamism" constraints imply that the plan of sensors relocation could fail at any time. For instance, a sensor which was moving to a safe place during a forest fire, could realize that fire has changed its direction and thus his destination is no longer safe.

Under the constraints cited above, the real environment is not necessary the best place to plan sensors relocation for the following reasons:

1. The relocation process needs the collaboration of several sensors which implies a high volume of exchanged messages between the physical sensors. Given the nature of the environment (natural disaster) and the commonly limited resources of sensors, such communication should be avoided.

2. The movement of each sensor strongly depends on different parameters, namely, the spatial characteristics of the surrounding space (elevation, slope, etc.), the evolution of the situation (e.g., fire progression in a forest), and the position of the other resources/sensors. These parameters are partially or totally unknown for individual sensors.

3. Individual sensors have only a partial vision of the situation. Each sensor is only aware of its neighborhood. Thus it lacks of the global vision of the situation, which is in this particular case necessary to find a global relocation strategy.

The artificial intelligence community has proposed solutions to similar problems through the Simulation-Based Planning approach (SBP) (Lee and Fishwick, 1994) which consists of associating planning and simulation. Each generated plan is simulated in order to be tested 
and assessed. The most appropriate plan is kept. However, when applied to real world problems, the current SBP approach does not suggest an efficient interaction between the real world and the simulated environment. In addition, monolithic planning turned out to be ineffective (Desjardins et al., 1999). Agent-based planning appeared then as a good alternative. Combining both techniques (simulation-based planning and agent-based planning) may then help to solve such problems. The SDSS which supports this idea should be able to build a synchronous parallel between the real world and the simulated environment (which is mainly the geo-referenced map) of the SDSS.

The sensor relocation application can be thought of as a layered architecture as illustrated in Fig. 2. This design philosophy is inspired by the layered simulation model we proposed in (Sahli and Moulin, 2009). The real sensors are of course located in the real world. Each of these sensors has a representative in the simulated environment. This representative is a software agent. Sensor relocation is thus planned by these agents in the SDSS and then communicated to the real sensors. The SDSS should include four layers as shown in Fig 2. A full description of these layers can be found in (Sahli and Jabeur, 2011).

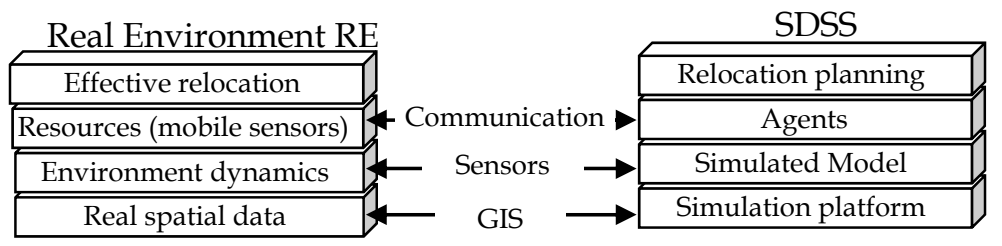

Fig. 2. Four-layer architecture of geosimulation based SDSS for sensors relocation

As the relocation planning takes place in the SDSS, the SDSS needs to be continuously updated by the real environment to maintain the coherency and synchronization between the two environments. In this context, the communication between the physical sensor and its agent representative is crucial. As for communication within the SDSS, heavier message traffic is to be exchanged between different representatives in order to: (i) inform each other about corresponding sensors status; (ii) check the status of others' sensors; (iii) coordinate relocation. Coordinating the relocation is not an individual decision. Indeed, a sensor having the intension to move must inform and get the approval of the surrounding sensors especially that the movement must be for the sake of achieving a common goal. Besides, all moves should be aligned with a strategic action which may be dictated by a human decision-maker.

An SDSS supporting these four layers of Fig. 2 offers the following advantages: (i) Most of the interaction messages between agents (sensors' representatives) are taking place in the SDSS (locally). Even a high traffic of exchanged messages will not really affect the performance of the relocation process; (ii) Since the relocation planning will be conducted by the representative agents in the SDSS, each agent will have access to all relevant data (spatial data of the surrounding space from GIS, the last evolution of the situation from the simulated environment, and the position of the other resources and sensors from the other co-existing agents). More details about the internal architecture of these agents can be found in (Sahli and Moulin, 2009); (iii) As all agents are in the SDSS, a human decision-maker can have a global idea about the current situation. Therefore, he/she can take part in the relocation planning process by dictating strategic actions. 
Moreover, planning in a highly dynamic environment using a simulation-based approach implies dealing with another problem: when should the simulation process be launched in the SDSS and how the simulation process should be coupled with the effective relocation? Early planning applications were based on the assumption that a planner's environment is stable and that it is possible to create a complete plan before executing it (Russel and Norvig, 1995). Such an assumption is inappropriate when considering dynamically changing environments. Instead, it is necessary to adopt a continual planning $(\mathrm{CP})$ approach in which the activities of planning and execution are interleaved and in which re-planning is triggered by changes in the environment or when the executed actions abort (AmbrosIngerson and Steel, 1988). Nevertheless, deciding when and how to interleave planning and execution is one of the most complex problems of CP. In this context, we have already proposed a dedicated planning approach, named Anticipated Continual Planning (ACP) in (Sahli and Moulin, 2009). Our ACP is based on the Continual Planning paradigm but enhanced by a preventive re-planning step (anticipating and periodic) and by a corrective one (following an unexpected event). In both cases, a global plan is elaborated in parallel and by both software agents and human decision-makers. Our approach enhances the interleaving process of the classical CP (agents are not forced to stop the planning process in order to wait for the execution), increases the quality of the plan (the plan is periodically updated and eventual problems are anticipated and taken into account before having repercussion on the plan), and gives more guarantees about reaching a final solution (since a global plan is always available and in which human decision-makers have taken part).

\section{Towards better efficiency in sensor network-based SDSS}

As we previously mentioned, geospatial semantics is currently an active research topic which would resolve the problem of interoperability between different SDSS using heterogeneous spatial data sources and formats. Geospatial semantics is also very valuable for improving the efficiency of SDSS as this would help in providing the decision-makers with the right data, in the right format, at the right time. In this section we argue this idea and illustrate it with two of our previous works on sensor network-based SDSS and geosimulation-based SDSS.

\subsection{Semantic aspects for better efficiency in SDSS}

Maximizing the benefits from a sensor network-based SDSS would require to feed the SDSS with the right data, in the right format, from the right locations, with the right sensors, at the right time. This could be reached by a thorough examination of data semantics. Data semantics could help in delimiting the sensing areas and sensing resources (Jabeur and Haddad, 2009; Jabeur et al., 2008). In addition to preserving the sensor network resources, this would speed up getting data from the network.

Once data is pulled out from the sensor network, we can maximize the benefits from the semantics of geo-information by finding effective methods to: (1) encode data for different purposes, and (2) transform this data from one representation to another. The use of the appropriate data representation at the right time would improve the analysis of the current situation, pose the right queries to the network, increase data sharing, and reduce the consumption of the limited resources (Jabeur and Haddad, 2009).

Several approaches can be used for data representation in sensor network while increasing the benefits from the semantics of data. Semantic networks (Lehmann, 1992) and Frames 
(Minsky, 1974) are examples of these approaches. The use of the XML and GML (Cox, 2006) languages provides a standard and common ways of cataloguing and exchanging sensor network information. The Open Geospatial Consortium (OGC) proposes the Observations and Measurements (O\&M) standard (Cox, 2006) that defines a conceptual schema and XML encoding for observations and for features involved in sampling when making observations. OGC also proposes the Sensor Observation Service (SOS) (Na and Priest, 2006) standard that defines a web service interface for the discovery and retrieval of real time or archived data produced by all kinds of sensors. By using GML, O\&M documents can be generated in SOS software. In this case, measurement records are stored to a database with a schema based on the O\&M specification. When this database is queried, the SOS server pulls the relevant records from the database and automatically generates the O\&M document in XML format based on a template that is stored on the server (McCarthy, 2007).

\subsection{Encoding data using conceptual graphs}

Knowledge about the semantic of hazardous events and their behaviours are extremely valuable for developing SDSS that can predict situations evolution and prevent dangerous consequences before they occur. For example, rising water levels indicate that flooding may be occurring, and the sudden motion of a slope can signify a slope failure. Due to complex chain reactions of concurrent events, there is an urgent need to automate the sensor network activities while preventing the waste of its limited resources. The representation of available data in the appropriate format and the encoding of expert knowledge in a machine-usable form serve to reinforce any decision support that a monitoring system may draw.

In (Jabeur and Haddad, 2009) authors proposed an approach that takes benefit from the semantics of data on natural phenomena conceptualized as spatio-temporal events and formalised using conceptual graphs (CGs). Based on Peirce's existential graphs and semantic networks of artificial intelligence, CGs were introduced by Sowa (Sowa, 1984) and provide an extensible system of logic to capture, represent and reason with the semantic of real-world knowledge. The approach proposed in (Jabeur and Haddad, 2009) uses causal knowledge about hazardous events and their effects in time and space to implement a progressive hazard monitoring. Hazardous events (events of interest, such as flood and rain fall) are explicitly conceptualised as static states and dynamic changes (punctual and durative) which are delimited in space and time (Haddad and Moulin, 2010a). The effects of a given hazardous event are formalised using causality relationships that define semantic, spatial and temporal causal constraints between cause and effect situations. These constraints are derived from the fact that human recognition of causal relations is based upon recognition of precedence and contiguity between the cause and the effect (Kitamura et al., 1997). Knowledge about hazardous events, their effects and risk levels were formalised using CGs and used to improve the efficiency of the sensor-network. When an event of interest is triggered by the sensors, the system first identifies what are its possible effects, their associated level risk, and where and when they may occur and then reserves the relevant sensor network resources in order to collect data in right place and time (Jabeur and Haddad, 2009).

In a related work (Mekni and Haddad, 2010) authors used semantic knowledge about events and their spatio-temporal effects to develop an integrated knowledge-based multi-agent geo-simulation framework for intelligent sensor web deployment. Figure 3 illustrates the main components of the framework. 


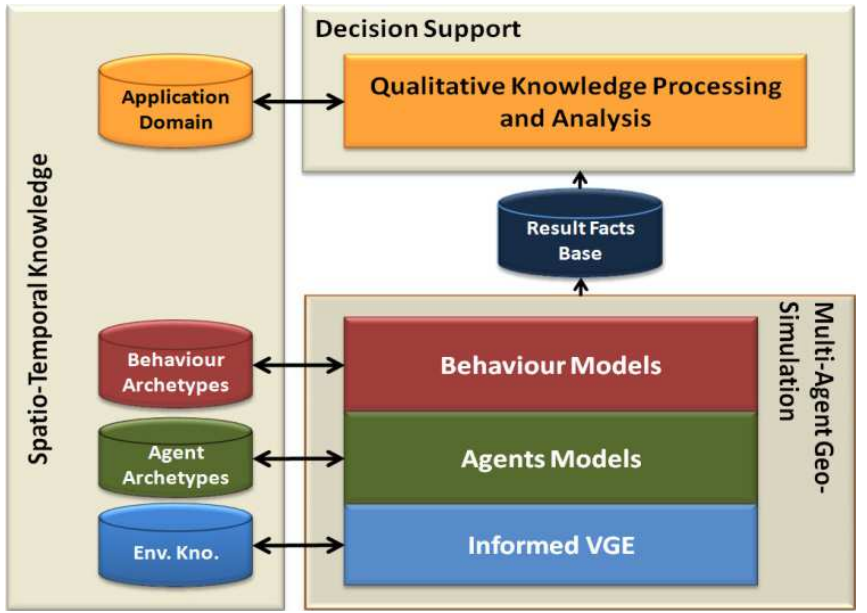

Fig. 3. Architecture of the knowledge-based multi-agent geo-simulation framework for intelligent sensor web deployment (Mekni and Haddad, 2010)

Multi-Agent Geo-Simulation is used to simulate the behaviour of a sensor network in a dynamic virtual geographic environment. Sensors are modeled as intelligent agents embedded in an informed virtual space where dynamic phenomena can occur. Sensor agents have reasoning capabilities allowing them to reason about the virtual space and to react to its dynamic phenomena. They have perception ranges and can be active or sleeping. Spatio-Temporal knowledge encoded in CGs is used for two main purposes. First, it is used during the geosimulation to support sensor agents reasoning capabilities. In the beginning of the simulation only some sensor agents are active. When an active sensor agent detects the occurrence of a situation of interest (such as a storm) in its perception range, it asks the knowledge base for its consequences, delimits when and where they may occur and then wakes up the relevant sleeping sensors within certain distance. Once the situation of interest is out the perception range of the sensor agent it switches to the sleeping mode. Second, the Spatio-temporal knowledge is used to analyse the results of the geo-simulation and to offer decision support to users (evaluation of different sensor deployment plans).

\section{Conclusion}

When considering any DSS, it is very important to know the degree of involvement of human experts in the process of taking decisions. This issue is more crucial during crisis response, especially when decisions are to be taken in real-time. As the spatial component arises, the trust of decision-makers in SDSS needs a longer discussion. In this chapter, we discussed briefly the problem from the cognitive point of view. A longer investigation supported by a real example can be found in (Sahli and Moulin, 2006). The cognitive aspect helps in understanding the limits of SDSS and pinpointing the issues where human decision-makers could not be overlooked.

When dealing with geographic reasoning, which is typically based on incomplete information, a human planner is able to draw quite accurate conclusions by cleverly completing the information or applying certain default rules based on common sense. Despite these cognitive capabilities, the planner has limitations when it comes to simulate complex events, particularly 
in a real large-scale geographic space. This task could be achieved properly by an SDSS. While the SDSS "knows" better the spatial environment as it uses GIS data, it does not have a refined sense of anticipation and judgment as a human expert does. Even if a plan generated by the SDSS seems to be well-grounded, it may not be feasible in reality or may go against certain doctrines. Human experts can propose to adjust/change the SDSS' recommendations according to their own experience and anticipation sense. For these reasons, we draw the conclusion that geosimulation based SDSS complements human planning skills when addressing complex problems such as crisis situations. It is worthwhile to investigate this degree of complementarities and come up with some general guidelines that could be customized depending on the application domain.

\section{Acknowledgement}

The research leading to these results has received Research Project Grant Funding from the Research Council of the Sultanate of Oman Research Grant Agreement No [ORG DU ICT 10 003]

\section{References}

Agatsiva, J., \& Oroda, A. (2002). Remote sensing and GIS in the development of a decision support system for sustainable management of the drylands of eastern Africa: A case of the Kenyan drylands, International Archives of the Photogrammetry, Remote Sensing and Spatial Information Sciences, 34:42-49.

Albrecht, J. (2007). Dynamic GIS, in The Handbook of Geographic Information Science, ed. J. Wilson and S. Fotheringham, London: Blackwell, pp. 436-446.

Ambros-Ingerson J.A. Steel, S. (1988). Integrating planning, execution and monitoring." In Proc. of the $7^{\text {th }}$ (US) National Conference on Artificial Intelligence (AAAI 88), St Paul, MN, USA. Americain Association for Artificial Intelligence, Morgan Kaufmann, pp. 83-88

Botts, M., Percivall, G., Reed, C., Davidson, J. (2006). OGC Sensor Web Enablement: Overview and High Level Architecture. Open Geospatial Consortium Whitepaper. Open Geospatial Consortium.

Cárdenas Tamayo, R.A; Lugo Ibarra, M.G. and Garcia Macias, J.A. (2010): Better crop management with decision support systems based on wireless sensor networks, Electrical Engineering Computing Science and Automatic Control (CCE), 2010 7th International Conference on, 2010, 412 - 417, Tuxtla Gutierrez

Chien, S.; Tran, D.; Doubleday, J.; Davies, A.; Kedar, S.; WEbb, F.; Rabideau, G.; Mandl, D.; Frye, S.; Song, W.; Shirazi, B. and Lahusen, R. (2010). A Multi-agent Space, In-situ Volcano Sensorweb, International Symposium on Space Artificial Intelligence, Robotics, and Automation for Space (i-SAIRAS 2010). Sapporo, Japan. August 2010

Cox, S. (2006). Observations and measurements. OpenGIS Discussion Paper. Open Geospatial Consortium (2006)

Craik, K.J.W. (1943). The Nature of Explanation. London: Cambridge. University Press, 1943.

Cutter, S. (1993). Living with Risk: The Geography of Technological Hazards, London: Edward Arnold.

Densham, P. J., Goodchild, M. F. (1989). Spatial Decision Support System: A Research Agenda. Proceedings of GIS/LIS '89, Florida, pp. 707-716.

Densham, P.J. (1991). Spatial decision support systems. In: D.J. Maguire, M.F. Goodchild, D.W. Rhind (eds.) Geographical information systems: principles and applications, pp. 403-412. London: Longman.

Desjardins, M.; Durfee, E.; Ortiz, C. and Wolverton, M. (1999). A survey of research in distributed continual planning. AI Mag. vol. 4, 1999, pp. 13-22. 
Evers, M. (2007). Requirements for Decision Support in Integrated River Basin Management, Journal for Management of Environmental Quality, Vol. 19(1), 2007

Fedra, K. \& Reitsma, R.F. (1990). Decision Support and Geographical Information Systems. In Geographical Information Systems for Urban and Regional Planning, Research Report: RR-90-009. Laxenburg, Austria: ACA, International Institute for Applied Systems Analysis (IIASA).

Fedra, K. (1997). Integrated risk assessment and management: Overview and state-of-theart, Journal of Hazardous Materials, 61:5-22.

Filippoupolitis, A. \& Gelenbe, E. (2009). A distributed decision support system for building evacuation. In Proceedings of the 2nd conference on Human System Interactions (HSI'09). IEEE Press, Piscataway, NJ, USA, 320-327

Forbus, K.D. (1981). A Study of Qualitative and Geometric Knowledge in Reasoning about Motion, MIT Artificial Intelligence Laboratory, Research Report AI-TR-615, 1981.

Fulcher, C.; Prato, T.; Vance, S.; Zhou, Y. and Barnett, C. (1995). Flood impact decision support system for St. Charles, Missouri, USA, 1995 ESRI International User Conference, 22-26 May, Palm Springs, California, pp. 11.

Gao, S., D. Sundaram, and J. Paynter. (2004). Flexible support for spatial decision making. Paper presented at the 37th Annual Hawaii International Conference on System Sciences, Honolulu, Hawaii.

Garbolino, V.; Sacile, R.; Olampi, S.; Bersani, C.; Alexandre, N.; Trasforini, E.; Benza, M. and Giglio, D. (2007). A Spatial Decision Support System prototype for assessing road HAZMAT accident impacts on the population in a dense urban area: a case study of the city of Nice, French Riviera, Proceedings of the eight International Conference on Chemical \& Process Engineering, Ischia, 24-27 June 2007.

Goel, R. K. 1999. Suggested framework (along with prototype) for realizing spatial decision support systems (SDSS). Paper presented at Map India 1999 Natural Resources Information System Conference, New Delhi, India.

Haddad, H. \& Moulin, B. (2007). Using Cognitive Archetypes and Conceptual Graphs to Model Dynamic Phenomena in Spatial Environments, In: ICCS 2007, LNAI 4604, U. Priss; S. Polovina \& R. Hill (Eds.), pp. 69-82, Springer-Verlag, Berlin Heidelberg

Haddad, H. \& Moulin, B. (2010a). A Framework to Support Qualitative Reasoning about COAs in a Dynamic Spatial Environment. International Journal of Experimental $\mathcal{E}$ Theoretical Artificial Intelligence, Vol. 22(4), pp. 341 - 380, Taylor \& Francis

Haddad, H. \& Moulin, B. (2010b). Multi-Agent Geo-Simulation in Support to Qualitative Spatio-Temporal Reasoning, Modeling Simulation and Optimization - Focus on Applications, Shkelzen Cakaj (Ed.), pp. 159-184, ISBN: 978-953-307-055-1, INTECH

Hoc J. M. (1987). Cognitive Psychology of Planning. Academic press, 1987.

Jaber, A.; Guarnieri, F. and Wybo, J.L. (2001). Intelligent software agents for forest fire prevention and fighting, Safety Science, 39, pp. 3-7.

Jabeur, N. \& Haddad, H. (2009). Using Causality Relationships for a Progressive Management of Hazardous Phenomena with Sensor Networks. In proceedings of the International Conference on Computational Science and Applications (ICCSA 2009), June 29-July 2, 2009, Yongin, Korea, pp: 1-16

Jabeur, N.; McCarthy, J.D. and Graniero, P. (2008). Improving Wireless Sensor Network Efficiency and Adaptability through an SOS Server Agent. In proceeding of the $1^{\text {st }}$ IEEE International workshop on the Applications of Digital Information and Web Technologies (ICADIWT 2008). 4-6 August, Ostrava, Czech Republic, pp: 409-414.

Jensen, J.R.; Hodgson, M.E.; M. G-Q Quijano, J. Im.(2009). A Remote Sensing and GIS-assisted Spatial Decision Support System for Hazardous Waste 
Site Monitoring, Photogrammetric Engineering and Remote Sensing, 75(2): 169-177.

Kahneman, D. \& Tversky, A. (1982). The simulation heuristic. In Kahneman, Slovic \& Tversky (Eds.) Judgement under uncertainty: Heuristics and biases. New York: Cambridge University Press, 1982.

Keenan, P. B. (2003). Spatial Decision Support Systems, in M. Mora, G. Forgionne, and J. N. D. Gupta (Eds.), Decision Making Support Systems: Achievements and challenges for the New Decade: Idea Group, pp. 28-39.

Keramitsoglou, I.; Kiranoudis, C. T.; Sarimveis, H. and Sifakis, N. (2004). A multidisciplinary decision support system for forest fire crisis management. Environmental management, vol 33, No 2, pp 212-225

Kitamura, Y.; Ikeda, M. and Mizoguchi, R. (1997). A Causal Time Ontology for Qualitative Reasoning. In: The Fifteenth International Joint Conference on Artificial Intelligence IJCAI 1997, pp. 501-506 (1997)

Kitchin, R. M. (1994). Cognitive Maps: What Are They and Why Study Them?, Journal of Environmental Psychology, 1994, pp. 14:119.

Lee, J.J. \& Fishwick, P.A. (1994). Real-Time Simulation-Based Planning for Computer Generated Force Simulation, Simulation, vol. 63, n 5, 1994, pp. 299-315.

Lehmann, F. (1992). Semantic networks. Computers and Mathematics with Application, 23 (2-5), 1-50, (1992)

Ling, B.; Zeifman, M.; Traweek, M. and Wettergren, T. (2007). A Sparse Undersea Sensor Network Decision Support System Based on Spatial and Temporal Random Field, Unattended Ground, Sea, and Air Sensor Technologies and Applications IX. Edited by Carapezza, Edward M.. Proceedings of the SPIE, Volume 6562, pp. 65620P (2007).

MacLean, D.A.; MacKinnon, W.E.; Porter, K.B.; Beaton, K.P.; Cormier, G. and Morehouse, S. (2000). Use of forest inventory and monitoring data in the spruce budworm decision support system, Computers and Electronics in Agriculture, 28:101-118.

Malczewski, J. 1999. GIS and multicriteria decision analysis. New York: John Wiley \& Sons, Inc.

McCarthy, J.D. (2007). Representing spatial and domain knowledge within a spatial decision support framework. M.Sc. Thesis, University of Windsor (2007)

McCarthy, J.D.; Graniero, P.A. and Rozic, S.M. (2008). An integrated GIS-expert system framework for live hazard monitoring and detection. Sensors 8: 830-846.

Mekni, M. and Haddad, H. (2010). A Knowledge-Based Multi-Agent Geo-Simulation Framework: Application to Intelligent Sensor Web Deployment, Proceedings of the fourth International Conference on Sensor Technologies and Applications, SENSORCOMM 2010, July 18-25, 2010 - Venice / Mestre, Italy, pp.329-335, ISBN: 978-0-7695-4096-2

Miller, R.; Guertin, D.P. and Heilman, P. (2003). An Internet-based Spatial Decision Support System for Rangeland Watershed Management." First Interagency Conference on Research in the Watersheds: October 27-30, 2003. U.S. Dept. of Agriculture, Agricultural Research Service, p. 725-730.

Minsky, M. (1974). A framework for representing knowledge. Cambridge, MA, USA: Massachusetts Institute of Technology, (1974)

Na, A., Priest, M. (2006). OpenGIS Sensor Observation Service Implementation Specification. Open Geospatial Consortium

Nisha de Silva, F. (2000). Challenges in Designing Spatial Decision Support Systems for Evacuation Planning, Natural Hazards Research paper, 2000

O’Brien, W.; Julien, C.; Kabadayi, S; Luo, X. and Hammer, J. (2009). An Architecture for Decision Support in ad hoc Sensor Netwoks, Information Technology in Construction, Vol. 14, 2009, pg. 309 
Ozan, Erol \& Kauffmann, Paul (2002). A Simulation-based Spatial Decision Support System for a New Airborne Weather Data Acquisition System, 2002 Industrial Engineering Research Conference, May 19-22, 2002, Orlando, Florida

Radke, J. (1995). A spatial decision support system for urban/wildland interface fire hazards, 1995 ESRI International User Conference, 22-26 May, Palm Springs, California, pp. 14.

Rozic, S. M. (2006). Representing spatial and domain knowledge within a spatial decision support framework. M.Sc. Thesis, University of Windsor (2006)

Russel, S. \& Norvig P. (1995). Artificial Intelligence, a Modern Approach, Prentice Hall, 1995.

Sahli, N. \& Jabeur, N. (2011). Agent-Based Approach to Plan Sensors Relocation in a Virtual Geographic Environment" in Proceedings of NTMS 2011, Paris, France, 7-10 February 2011.

Sahli, N. \& Moulin, B. (2006). Agent-based geo-simulation to support human planning and spatial cognition (extended version). J.S. Sichman and L. Antunes (Eds.): MABS 2005, Springer-Verlag Berlin Heidelberg LNAI 3891, pp. 115 - 132, 2006.

Sahli, N. \& Moulin, B. (2009). EKEMAS, an agent-based geo-simulation framework to support continual planning in the real-word" [Quick Edit] Applied Intelligence, Vol. 31, No. 2. (1 October 2009), pp. 188-209.

Sahli, N.; Mekni, M. and Moulin, B. (2008). A Multi-Geosimulation Approach for the Identification of Risky Areas for Trains, Workshop Agents in Traffic and Transportation, part of the International Conference AAMAS 2008, Portugal, 2008.

Sanders, R., \& Tabuchi, S. (2000). Decision support system for flood risk analysis for the River Thames, United Kingdom, Photogrammetric Engineering \& Remote Sensing, 66(11), pp. 1185-1193.

Segrera, S.; Ponce-Hernandez, R. and Arcia, J. (2003). Evolution of decision support system architectures: Applications for land planning and management in Cuba. Journal of Computer Science \& Technology 3(1), pp. 40-46.

Sowa, J.F. (1984). Conceptual Structures: Information Processing in Mind and Machine. Addison-Wesley, Massachusetts (1984)

Sprague, R. H. and H. J. Watson (1996). Decision support for management. Upper Saddle River, N.J.: Prentice Hall

Sugumaran, R. \& Degroote, J. (2010). Spatial Decision Support Systems: Principles and Practices, CRC Press; 1 edition

Sugumaran, V., and Sugumaran, R. 2005. Web-based Spatial Decision Support Systems (WebSDSS): Evolution, Architecture, and Challenges, Third Annual SIGDSS PreICIS Workshop, Designing Complex Decision Support: Discovery and Presentation of Information and Knowledge, Las Vegas, Nevada (USA).

Uran, O. \& Janssen, R. (2003). Why are spatial decision support systems not used? Some experiences from the Netherlands. Computers, Environment and Urban Systems 27(5), pp. 511-526

Wang, Y, Gong, J. and Wu, X. (2007). Geospatial semantic interoperability based on ontology, GEO-SPATIAL INFORMATION SCIENCE, Vol 10(3), pp. 204-207

Wicklet, G. \& Potter, S. (2009). Information-gathering: From sensor data to decision support in three simple steps, Intelligent Decision Technologies, Vol. 3(1), 2009, pp. 3-17

Zhang, C. (2010). Develop a Spatial Decision Support System based on Service-Oriented Architecture, Decision Support Systems, Chiang S. Jao (Ed.), ISBN 978-953-761964-0, pp. 406, January 2010, INTECH, Croatia

Zhang, M. \& Grant, P.W. (1993). Pages 107-114 in Proceedings of the Thirteenth Annual ESRI User Conference, May 1993. Redlands, California: Environmental Systems Research Institute. 


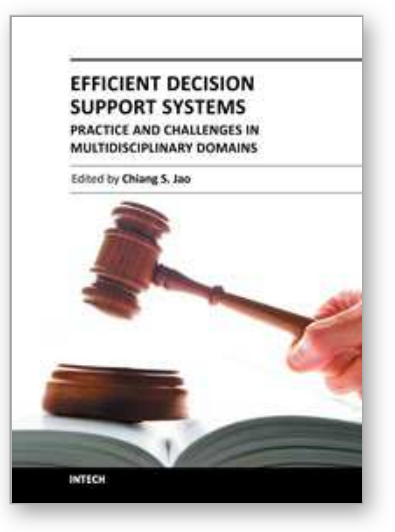

\author{
Efficient Decision Support Systems - Practice and Challenges in \\ Multidisciplinary Domains \\ Edited by Prof. Chiang Jao
}

ISBN 978-953-307-441-2

Hard cover, 478 pages

Publisher InTech

Published online 06, September, 2011

Published in print edition September, 2011

This series is directed to diverse managerial professionals who are leading the transformation of individual domains by using expert information and domain knowledge to drive decision support systems (DSSs). The series offers a broad range of subjects addressed in specific areas such as health care, business management, banking, agriculture, environmental improvement, natural resource and spatial management, aviation administration, and hybrid applications of information technology aimed to interdisciplinary issues. This book series is composed of three volumes: Volume 1 consists of general concepts and methodology of DSSs; Volume 2 consists of applications of DSSs in the biomedical domain; Volume 3 consists of hybrid applications of DSSs in multidisciplinary domains. The book is shaped decision support strategies in the new infrastructure that assists the readers in full use of the creative technology to manipulate input data and to transform information into useful decisions for decision makers.

\title{
How to reference
}

In order to correctly reference this scholarly work, feel free to copy and paste the following:

Nafaâ Jabeur, Nabil Sahli and Hedi Haddad (2011). Sensor Network and GeoSimulation: Keystones for Spatial Decision Support Systems, Efficient Decision Support Systems - Practice and Challenges in Multidisciplinary Domains, Prof. Chiang Jao (Ed.), ISBN: 978-953-307-441-2, InTech, Available from:

http://www.intechopen.com/books/efficient-decision-support-systems-practice-and-challenges-inmultidisciplinary-domains/sensor-network-and-geosimulation-keystones-for-spatial-decision-support-systems

\section{INTECH}

open science | open minds

\section{InTech Europe}

University Campus STeP Ri

Slavka Krautzeka 83/A

51000 Rijeka, Croatia

Phone: +385 (51) 770447

Fax: +385 (51) 686166

www.intechopen.com

\section{InTech China}

Unit 405, Office Block, Hotel Equatorial Shanghai

No.65, Yan An Road (West), Shanghai, 200040, China

中国上海市延安西路65号上海国际贵都大饭店办公楼405单元

Phone: +86-21-62489820

Fax: $+86-21-62489821$ 
(C) 2011 The Author(s). Licensee IntechOpen. This chapter is distributed under the terms of the Creative Commons Attribution-NonCommercialShareAlike-3.0 License, which permits use, distribution and reproduction for non-commercial purposes, provided the original is properly cited and derivative works building on this content are distributed under the same license. 